\title{
Impact of complex adatom-induced interactions on quantum spin Hall phases
}

\author{
Flaviano José dos Santos, ${ }^{1,2}$ Dario A. Bahamon, ${ }^{3}$ Roberto B. Muniz, ${ }^{4}$ Keith McKenna, ${ }^{5}$ Eduardo V. Castro,,${ }^{6,7}$ \\ Johannes Lischner, ${ }^{8, *}$ and Aires Ferreira ${ }^{5, \dagger}$ \\ ${ }^{1}$ Peter Grünberg Institut and Institute for Advanced Simulation, Forschungszentrum Jülich \& JARA, D-52425 Jülich, Germany \\ ${ }^{2}$ RWTH Aachen University, D-52056 Aachen, Germany \\ ${ }^{3}$ MackGraphe - Graphene and Nano-Materials Research Center, Mackenzie Presbyterian University, Rua da Consolação 896, \\ 01302-907, São Paulo, SP, Brazil \\ ${ }^{4}$ Instituto de Física, Universidade Federal Fluminense, Niterói, Brazil \\ ${ }^{5}$ Department of Physics, University of York, York YO10 5DD, United Kingdom \\ ${ }^{6}$ CeFEMA, Instituto Superior Técnico, Universidade de Lisboa, Avenida Rovisco Pais, P-1049-001 Lisboa, Portugal \\ ${ }^{7}$ Beijing Computational Science Research Center, Beijing 100084, China \\ ${ }^{8}$ Department of Physics and Department of Materials, and the Thomas Young Centre for Theory and Simulation of Materials, \\ Imperial College London, London SW7 2AZ, United Kingdom
}

(Received 21 December 2017; published 17 August 2018)

\begin{abstract}
Adsorbate engineering offers a seemingly simple approach to tailor spin-orbit interactions in atomically thin materials and thus to unlock the much sought-after topological insulating phases in two dimensions. However, the observation of an Anderson topological transition induced by heavy adatoms has proved extremely challenging despite substantial experimental efforts. Here, we present a multiscale approach combining advanced first-principles methods and accurate single-electron descriptions of adatom-host interactions using graphene as a prototypical system. Our study reveals a surprisingly complex structure in the interactions mediated by random adatoms, including hitherto neglected hopping processes leading to strong valley mixing. We argue that the unexpected intervalley scattering strongly impacts the ground state at low adatom coverage, which would provide a compelling explanation for the absence of a topological gap in recent experimental reports on graphene. Our conjecture is confirmed by real-space Chern number calculations and large-scale quantum transport simulations in disordered samples. This resolves an important controversy and suggests that a detectable topological gap can be achieved by increasing the spatial range of the induced spin-orbit interactions on graphene, e.g., using nanoparticles.
\end{abstract}

DOI: 10.1103/PhysRevB.98.081407

The attachment of adsorbates to two-dimensional materials has attracted much interest in recent years, as a route to tailoring material properties and realizing novel phenomena [1-5]. In graphene, adatoms have been shown to induce band gaps [6-10], magnetic moments [11-13], and even superconductivity [14-17].

Adsorbate engineering could likewise provide atomic control over fundamental spin-orbit phenomena, such as spin relaxation [18-21] and Mott (skew) scattering [22-25]. Recent studies have predicted that the dilute assembly of heavy adatoms can massively enhance the weak spin-orbit energy gap of graphene [26-29], opening a promising route towards the realization of nontrivial topological insulating phases, including the quantum spin Hall (QSH) state [30]. However, transport measurements on samples decorated with heavy species, including In and Ir, have yet to show any signature of topological gap opening [31-35]. In this Rapid Communication, we show that a thorough treatment of disordercombining accurate model Hamiltonians with quantum transport simulations-is essential to predict the topological character of adatom-engineered systems and reconcile this contradiction. Our approach reveals that randomly distributed

\footnotetext{
*jlischner597@gmail.com

†aires.ferreira@york.ac.uk
}

heavy adatoms on graphene give rise to scattering between inequivalent valleys in the band structure, hindering the emergence of topologically protected edge states even in the absence of extrinsic factors, such as adatom clustering [36]. This resolves a controversy regarding the nature of spin-orbit interactions in adatom-decorated graphene and indicates that decoration with small clusters or nanoparticles, for which intervalley scattering is strongly reduced, may offer a route towards the realization of the much sought-after QSH phase.

Pristine graphene is a QSH insulator, but the smallness of its intrinsic spin-orbit interaction (in the range of $25-50 \mu \mathrm{eV}$ [37-40]) has thus far precluded achieving the dissipationless quantum transport regime [41-43]. The opening of a detectable QSH gap requires a massive enhancement of graphene's characteristic spin-orbit coupling (SOC), which preserves spin angular momentum $S_{z}$ [30]. Previous work suggested that this can be achieved via decoration with nonmagnetic adatoms with a $p$-outer electron shell [26], as they induce spin-conserving 'intrinsiclike' SOC [22]. As a prototypical heavy element, we consider thallium (Tl) [26]. The quasiparticle band structure of the thallium adatom on graphene was obtained employing a fully relativistic ab initio $G W$ approach; see Supplemental Material (SM) [44] for details.

First-principles multiscale approach. We use our first-principles supercell calculations to parametrize a 
single-electron Hamiltonian capturing all the relevant interactions mediated by (dilute) adatoms embedded in large area graphene samples. The first step is to derive a graphene-single-adatom tight-binding (TB) model that faithfully reproduces the $a b$ initio band structure. Enforcing time-reversal symmetry and invariance with respect to the $C_{6 v}$ point group, one easily finds $H=H_{g}+H_{a}+V_{g a}[22,26]$, where

$$
\begin{gathered}
H_{g}=-t \sum_{\langle i j\rangle} c_{\boldsymbol{r}_{i}}^{\dagger} c_{\boldsymbol{r}_{j}}+\left(\delta H_{t^{\prime}, t^{\prime \prime}}-\delta \mu \sum_{i \in P} c_{\boldsymbol{r}_{i}}^{\dagger} c_{\boldsymbol{r}_{i}}\right) \\
H_{a}=\sum_{m=0, \pm 1} \epsilon_{|m|} d_{m}^{\dagger} d_{m}+\lambda\left(d_{1}^{\dagger} s^{z} d_{1}-d_{-1}^{\dagger} s^{z} d_{-1}\right) \\
+\sqrt{2} \lambda\left(d_{0}^{\dagger} s^{-} d_{-1}+d_{0}^{\dagger} s^{+} d_{1}+\text { H.c. }\right) \\
V_{g a}=-\sum_{m=0, \pm 1}\left(i^{|m|} \tau_{|m|} \Omega_{m}^{\dagger} d_{m}+\text { H.c. }\right) .
\end{gathered}
$$

The first two terms are the Hamiltonians of $\pi$ electrons on graphene and $6 p$ states of a $\mathrm{Tl}$ atom, respectively. $c_{\boldsymbol{r}_{i}}^{\dagger} \equiv\left(c_{\boldsymbol{r}_{i} \uparrow}^{\dagger}, c_{\boldsymbol{r}_{i} \downarrow}^{\dagger}\right)$ and $d_{m}^{\dagger} \equiv\left(d_{m \uparrow}^{\dagger}, d_{m \downarrow}^{\dagger}\right)$ are the corresponding fermionic creation operators, $s^{x, y, z}$ are Pauli matrices acting on the spin space, and $s^{ \pm}=\left(s^{x} \pm i s^{y}\right) / 2$. The sites adjacent to the adatom define a hexagonal plaquette, $P \equiv\{1, \ldots, 6\}$. $V_{g a}$ is the adatom-graphene hybridization term written as a function of the plaquette operator for states with definite angular momentum $\Omega_{m}^{\dagger}=(1 / \sqrt{6}) \sum_{j \in P} \exp \{i \pi m(j-1) / 3\} c_{\boldsymbol{r}_{j}}^{\dagger}$ [66]. Next-nearest- and third-nearest-neighbor corrections $\left(\delta H_{t^{\prime}, t^{\prime \prime}}\right)$ are included in order to improve agreement to the first-principles results. The minimal Hamiltonian [Eqs. (1)(3)] contains nine parameters: the C-C hoppings $t, t^{\prime}$, and $t^{\prime \prime}$, the local chemical potential change on $\mathrm{C}$ sites next to $\mathrm{Tl}, \delta \mu$, the Tl outer-shell energies $\epsilon_{0}$ and $\epsilon_{ \pm 1}$, the Tl spin-orbit energy $\lambda$, and the C-Tl hoppings $\tau_{0}$ and $\tau_{ \pm 1}$. We adjust these parameters until the band structures quantitatively reproduce the firstprinciples calculations over a window of $\pm 1 \mathrm{eV}$ around the Dirac point (see SM [44]). The initial guess for the parameters is informed by a direct evaluation of hopping integrals between atom-centred maximally localized Wannier functions [44]. The quasiparticle band structure obtained from density functional theory (DFT)- and $G W$-parametrized minimal TB models is shown in Fig. 1. Bands below the Dirac point $(\epsilon \equiv 0)$ derive mostly from graphene $\pi$ states. The flatband with energy $\approx 0.4 \mathrm{eV}$ is a Tl $6 p$ state. Interestingly, the $G W$ corrections are seen to bring this band closer to the Dirac point. Moreover, the gap at the Dirac point is $23 \mathrm{meV}$ significantly larger than the DFT value of $13 \mathrm{meV}$. To what extent the optimistic first-principles estimates signal a measurable topological gap in real samples will depend on a delicate competition between spin-conserving SOC and two other interactions mediated by disorder, which we unveil in what follows.

Adatom scattering potential. In realistic conditions, dilute adatoms occupy random positions and thus act as scattering centers. The information on the adatom scattering potential is contained in the local density of states (LDOS) [68]. The crucial step in our multiscale approach consists of deriving a (graphene-only) TB model for the scattering potential compatible with the first-principles results for the
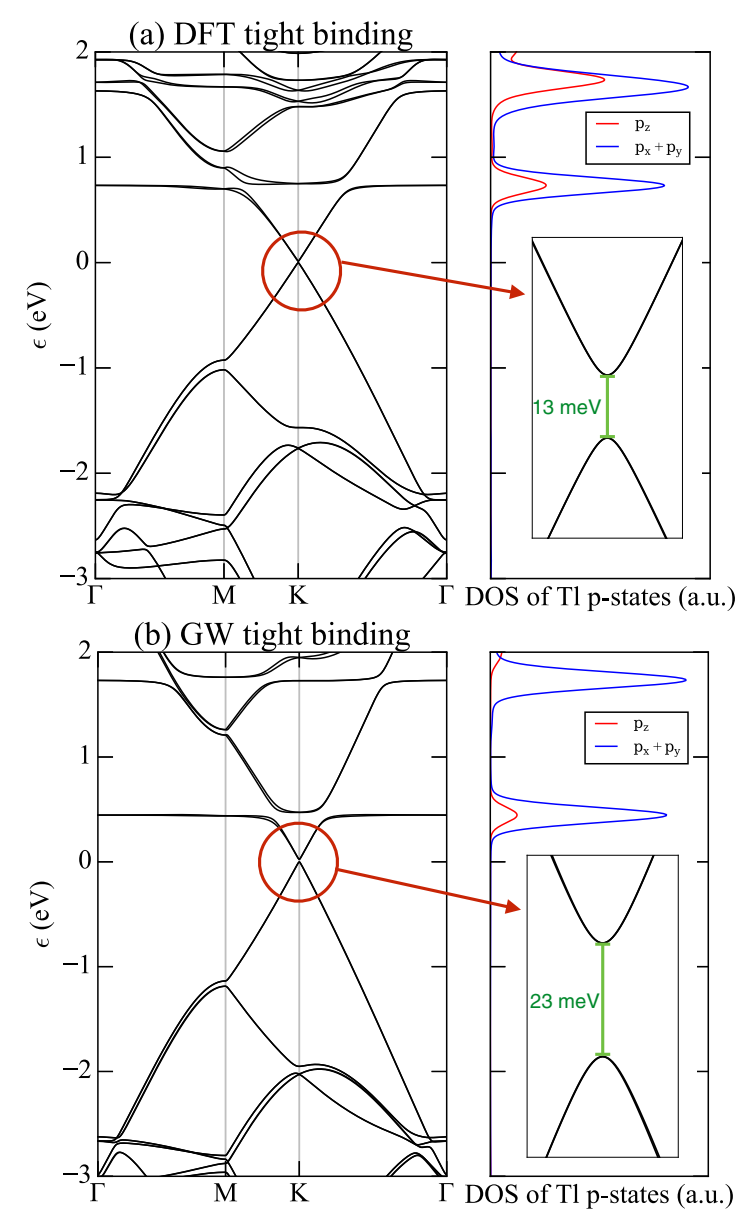

FIG. 1. (a) Fully relativistic DFT band structure for a thallium atom on graphene. (b) The corresponding quasiparticle band structure from a fully relativistic $G W$ calculation.

adatom-graphene supercell (see Fig. 1). To this end, we trace out the adatom degrees of freedom in Eqs. (1)-(3) through a Löwdin transformation. Formally, the resulting graphene-only TB Hamiltonian is given by $H_{\text {eff }}=H_{g}+\Sigma_{P}(\epsilon)$, where $\Sigma_{P}(\epsilon)$ is the real-space self-energy generated by a single adatom [44]. This term breaks translational symmetry and thus acts as a bona fide disorder interaction. Finally, the Hamiltonian of graphene with a dilute adatom coverage is obtained by adding up independent contributions $\left\{\Sigma_{P}(\epsilon)\right\}$ from adatoms located at random plaquettes $P_{k}(k=1 \ldots N)$. This procedure has two advantages. It captures all short-range interactions induced by the adatom (see below). Second, being based on a grapheneonly description, it allows a straightforward interpretation of quantum transport calculations. Explicit evaluation of the self-energy gives rise to the following effective interaction Hamiltonian $\hat{V}_{\text {dis }}=\sum_{k=1}^{N} \hat{\Sigma}_{P_{k}}$, with

$$
\begin{aligned}
\hat{\Sigma}_{P}= & \sum_{\langle i j\rangle \in P}\left(t_{1}^{\prime} c_{\boldsymbol{r}_{i}}^{\dagger} c_{\boldsymbol{r}_{j}}+\imath \zeta_{i j} t_{1}^{\prime \prime} c_{\boldsymbol{r}_{i}}^{\dagger} s^{z} c_{\boldsymbol{r}_{j}}\right) \\
& +\sum_{\langle\langle i j\rangle\rangle \in P}\left(t_{2}^{\prime} c_{\boldsymbol{r}_{i}}^{\dagger} c_{\boldsymbol{r}_{j}}+\imath \zeta_{i j} t_{2}^{\prime \prime} c_{\boldsymbol{r}_{i}}^{\dagger} s^{z} c_{\boldsymbol{r}_{j}}\right) \\
& +\sum_{\langle\langle i j\rangle\rangle \in P} t_{3}^{\prime} c_{\boldsymbol{r}_{i}}^{\dagger} c_{\boldsymbol{r}_{j}}+\lambda_{R} \sum_{i, j \in P} c_{\boldsymbol{r}_{i}}^{\dagger} \Lambda_{i j} c_{\boldsymbol{r}_{j}},
\end{aligned}
$$



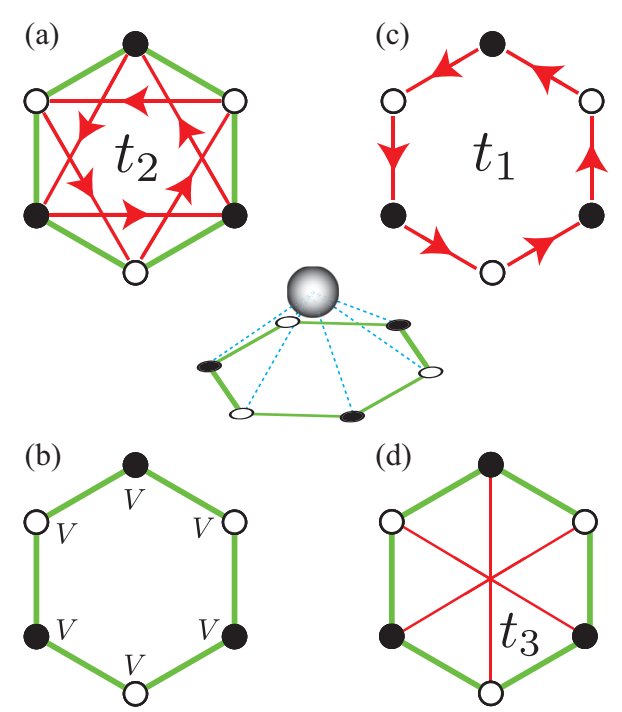

FIG. 2. Complex adatom-graphene interactions in realistic scenarios. (a) NNN hoppings. (b) On-site energies. (c), (d) Hopping processes opening the intervalley channel unveiled in this work. Green and red lines represent bare and adatom-induced hoppings, respectively; arrows indicate the presence of imaginary "chiral" components, making such hoppings sensitive to directions and spin projections.

where $\zeta_{i j}$ equals $\mp 1$ for circulation around the $P$ th plaquette (anti)clockwise and $\Lambda_{i j}=\imath\left(\xi_{i j} s^{+}+\xi_{i j}^{*} s^{-}\right)$with $\xi_{i j}=$ $\exp [l(j-1) \pi / 3]-\exp [l(i-1) \pi / 3]$. The effective hoppings are defined by $\lambda_{R}=\sqrt{2} \lambda \tau_{0} \tau_{1} / D_{\text {ad }}$ and

$$
t_{n}^{\prime}+\imath t_{n}^{\prime \prime}=e^{\imath n \pi / 3} X_{1}+X_{0}+e^{-\imath n \pi / 3} X_{-1},
$$

where $\quad X_{1}=\tau_{1}^{2}\left(\epsilon-\epsilon_{0}\right) / D_{\mathrm{ad}}, \quad X_{0}=\tau_{0}^{2}\left(\epsilon-\epsilon_{1}+\lambda\right) / D_{\mathrm{ad}}$, $X_{-1}=\tau_{1}^{2} /\left[6\left(\epsilon-\epsilon_{1}-\lambda\right)\right]$, and $D_{\text {ad }}=6\left[\epsilon^{2}-\epsilon\left(\epsilon_{0}+\epsilon_{1}-\right.\right.$ $\left.\lambda)+\epsilon_{0}\left(\epsilon_{1}-\lambda\right)-2 \lambda^{2}\right]$. The first terms $\left(t_{1}^{\prime}\right.$ and $\left.t_{1}^{\prime \prime}\right)$ modify the hopping between nearest-neighbor (NN) atoms, while the second line describes next-nearest-neighbor (NNN) hoppings, including a chiral component $\left(t_{2}^{\prime \prime}\right)$, which-in the absence of the other terms - transforms graphene into a QSH insulator [26]. The terms in the third line capture hoppings between $\mathrm{C}$ atoms on opposite sides of the adatom $\left(t_{3}\right)$ and spin-flip processes between all pairs of sites in the impurity plaquette $\left(\lambda_{R}\right)$. The latter is a Rashba-type interaction, which is vanishing small near the Dirac points and thus can be safely neglected $[23,26]$. The relevant interactions are visualized in Fig. 2.

Remarkably, the effective Hamiltonian obtained here by a rigorous adatom-decimation procedure is far more complex than previous models [26]. Importantly, $t_{1}^{\prime}$ and $t_{3}^{\prime}$ for heavy species can be significantly larger than the chiral NNN hopping. From the ab initio parameters derived for $\mathrm{Tl}$ [44] we obtain $t_{1}^{\prime} \approx 10 t_{2}^{\prime \prime}$ at $\epsilon=0$. To shed further light on the significance of hitherto neglected terms [Figs. 2(c) and 2(d)], we derive a long-wavelength effective description. As customary, we introduce the field operators $c_{\sigma \tau s}(\boldsymbol{k})=\int d \boldsymbol{r} e^{l(\boldsymbol{k}+\tau K) \cdot \boldsymbol{r}} \Psi_{\sigma \tau s}(\boldsymbol{r})$, with $\sigma[\tau]= \pm 1$ describing the projection low-energy states on the $A(B)$ sublattice (at $\boldsymbol{K}_{ \pm}$) for spin $s= \pm 1$. Substituting in Eq. (4) and performing a series expansion around the inequivalent Dirac points $\boldsymbol{K}_{ \pm}= \pm K \hat{k}_{x}$, one obtains the effective interaction $\hat{V}_{P}=\Psi^{\dagger}(\boldsymbol{r}) \hat{\mathcal{V}}_{P}(\boldsymbol{r}) \Psi(\boldsymbol{r})$ [69], with

$$
\hat{\mathcal{V}}_{P}(\boldsymbol{r})=\left(\Delta \tau_{z} \sigma_{z} s_{z}+g_{0} \tau_{x} \sigma_{x}+g_{1} \tau_{y} \sigma_{y} s_{z}\right) f_{P}(\boldsymbol{r}),
$$

to leading order in $\boldsymbol{k} / K$, and where we omitted a scalar term (see SM [44]). $\sigma$ and $\boldsymbol{\tau}$ denote Pauli matrices acting on sublattice and valley degrees of freedom, respectively, and $f_{P}(\boldsymbol{r}) \propto \delta\left(\boldsymbol{r}-\boldsymbol{r}_{P}\right)$ describes the spatial profile of the adatom potential. The first term derives from the NNN hopping $\Delta=3 \sqrt{3} t_{2}^{\prime \prime}[30]$. The remaining terms are scalar and spin-orbit interactions connecting valleys, with strengths $g_{0}=3\left(t_{3}^{\prime}-t_{1}^{\prime}\right)$ and $g_{1}=3 \sqrt{3} t_{1}^{\prime \prime}$, respectively. For $p$-outer-shell adatoms $t_{1}^{\prime \prime}=$ $t_{2}^{\prime \prime}$ [see Eq. (5)] and thus $g_{1}=\Delta$, i.e., intra- and intervalley spin-orbit scattering processes must be considered on equal footing. Based on general arguments for disordered zero-gap semiconductors, one expects that the mixing of states at inequivalent degeneracy points is detrimental for the topological phase [70-72]. An estimation using DFT-optimized parameters gives $g_{0}=0.41 \mathrm{eV}$ and $g_{1}=\Delta=-0.11 \mathrm{eV}$ at $\epsilon=0$. Such a dominance of intervalley processes in the coarsegrained description is a strong indication that the topological gap displayed by Tl-graphene supercells (Fig. 1) will be fragile in a disordered scenario, which would naturally explain the negative experimental results [31-35]. This idea is reinforced by the fact that electrons in graphene are very sensitive to valley mixing processes with an origin in short-range impurities, as the respective Friedel oscillations are known to decay as $1 / r$ at large distances (as opposed to the $1 / r^{2}$ law from intravalley scattering [68]).

Real-space quantum transport study. To investigate the implications of the complex structure of the effective adatom potential, we have carried out transport calculations within the Landauer-Büttiker framework. In the QSH regime, a pair of counterpropagating gapless edge states protected from elastic backscattering emerge at the interfaces to vacuum [Fig. 3(a)] [30]. To probe the robustness of the extrinsic QSH insulating phase and its concomitant helical edge states, we calculated the two-terminal conductance of large armchair nanoribbons (width $W=313.6 \mathrm{~nm}$, and length $L=$ $298.2 \mathrm{~nm}$ ) with randomly distributed adatoms connected to pristine graphene leads. The central channel contains in excess of 3.5 million atoms and efficient recursion techniques are employed to solve a system of this large size [44]. The smoking gun for the topologically protected edge states is the emergence of quantized conductance $G=2 e^{2} / h$ with a plateau width proportional to the SOC strength $[59,73]$. To probe the experimentally relevant adatom coverages would require prohibitively large computational domains, in order to resolve the typically small spin-orbit gaps $\Delta E \approx 0.3 n(\mathrm{eV})$, where $n$ is the adatom coverage. To overcome this difficulty, we rescaled uniformly the effective hoppings $t_{n} \rightarrow r t_{n}$ with $r=10$. The main findings are summarized in Fig. 3 . When only the intrinsic-type SOC term is included $\left(t_{2}^{\prime \prime}\right)$, the twoterminal conductance exhibits a plateau at small energies with $G=2 e^{2} / h$ [Fig. 3(b) (black dots)]. The variance $\Delta G$ is found to be zero up to numerical accuracy. Such a perfectly quantized effect shows that the nanoribbon has been transformed into a QSH insulator. The plateau width precisely saturates the upper bound $\Delta E_{\text {SOC }} \leqslant 2 n|\Delta|$, which is the topological gap obtained by Weeks et al. [26]. However, the plateau shrinks when $t_{1}^{\prime}$ is 

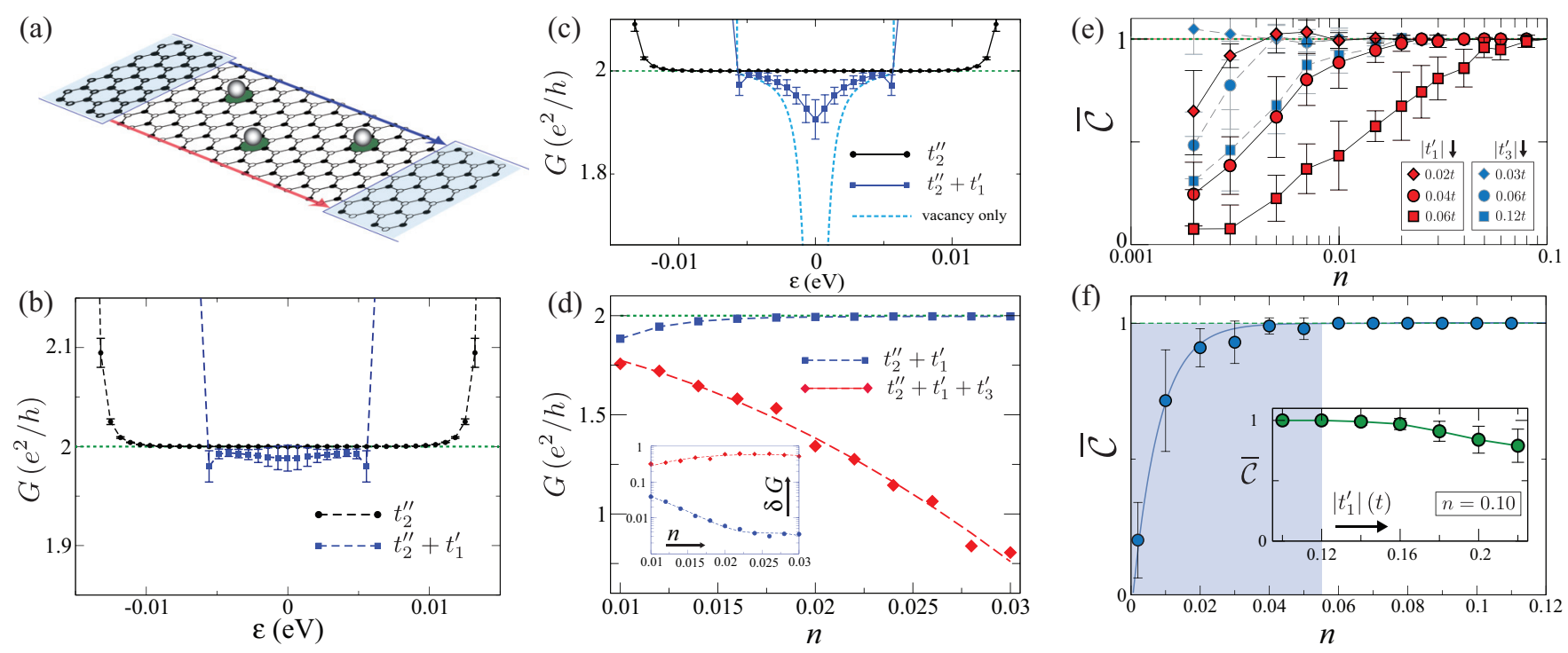

FIG. 3. Topological properties of nanoribbon and bulk graphene with random nonmagnetic heavy adatoms. (a) Schematic of a two-terminal device in the QSH regime; two edge states with opposite spins contribute with $G=2 e^{2} / h$. (b) Energy dependence of the conductance for two distinct adatom-graphene models ( $1 \%$ coverage). Parameters: $t=2.7 \mathrm{eV}, t_{1}^{\prime}=-2.4 \mathrm{eV}$, and $t_{2}^{\prime \prime}=-0.23 \mathrm{eV}$. (c) Conductance curve when a single vacancy is added to the adatom-decorated nanoribbon. Same parameters as in (b). The conductance of the defected nanoribbon in the absence of adatoms is also shown (dashed line). (d) Coverage dependence of the conductance showing the degradation of the quantized plateau. The behavior of fluctuations is shown in the inset. Parameters: Same as in (b) and (c) and $t_{3}^{\prime}=-1.0 \mathrm{eV}$. (e) Disorder-averaged Chern number as a function of adatom coverage for realistic spin-orbit coupling $\left(t_{2}^{\prime \prime}=0.01 t\right)$ and selected values of $t_{1}$ and $t_{3}$. (f) Coverage dependence of $\overline{\mathcal{C}}$ when all the interactions are "turned on" using DFT base values: $t_{1} / t=-0.12-0.01 i, t_{2}^{\prime} / t=-0.07-i 0.01$, and $t_{3} / t=-0.17$. Fitting to $\overline{\mathcal{C}}(n)=\tanh \left(n / n^{*}\right)$ yields a critical adatom coverage $n^{*} \approx 0.01$. The inset shows the dependence of $\overline{\mathcal{C}}$ with $t_{1}^{\prime}$ indicating a fast closing of the topological gap upon increasing $\mathrm{NN}$ hopping correction. Twenty-four independent adatom configurations were used in (b) and (c), and 100 in (d). For simplicity, adatom-induced hoppings are fixed to their values at the band center, $t_{n}(\epsilon=0)$, in all calculations.

turned on (blue dots), indicating a closing of the topological gap. This effect is accompanied by significative fluctuations $\Delta G \approx \pm 0.1 e^{2} / h$, showing that states delocalized through the nanoribbon contribute now to the electronic transport. This striking decay of helical edge states into the bulk is confirmed by the numerical evaluation of the spin-polarized bond current density maps; see SM [44]. The remaining adatom interactions affect the QSH phase in two different ways: (i) The imaginary $\mathrm{NN}$ hopping shifts the conductance plateau (not shown) and (ii) the hopping $t_{3}^{\prime}$ enlarges the central plateau and increases the fluctuations.

We now provide compelling evidence that QSH phases induced by random dilute adatoms are especially fragile in truly disordered scenarios, where additional sources of scattering are unavoidable. To this end, we introduce a topological defect (vacancy) in the nanoribbon, by cutting all bonds adjacent to one carbon atom. Vacancies introduce (quasi)localized states at zero energy strongly impacting the graphene electronic properties [74-79]. Given our choice of a metallic armchair nanoribbon, we locate the vacancy on a site with a finite density of states [80]. This choice guarantees that the vacancy works as a resonant scatter (introducing midgap states), leading to a strong suppression of the conductance at low energy, $G \rightarrow 0$ as $\varepsilon \rightarrow 0$ [Fig. 3(c)]. Adding a small coverage of idealized adatoms with only NNN hopping $\left(t_{2}^{\prime \prime}\right)$ gives rise to the expected quantization of the conductance, as the helical edge states resulting from the SOC enhancement can perfectly avoid the vacancy (no backscattering). Quite strikingly, when a NN hopping correction $t_{1}^{\prime} \approx 10 t_{2}^{\prime \prime}$ (typical of Tl adatoms in rows 5 and 6 of the periodic table) is turned on, the conductance acquires its basic shape prior to adatom decoration, unambiguously demonstrating the inherent fragility of the QSH phase due to the activation of intervalley processes. The dependence with the adatom coverage is shown in Fig. 3(d), which also shows the conductance at low energies is further degraded when $t_{1}^{\prime}$ and $t_{3}^{\prime}$ are taken simultaneously.

Chern number in real space. At this stage, we have firmly established that edge states in adatom-decorated graphene are intrinsically unstable due to hitherto neglected hoppings $t_{1}^{\prime}$ and $t_{3}^{\prime}$ (Fig. 2). As shown by our multiscale theory bridging advanced first-principles calculations and accurate TB models, such hoppings are typically one order of magnitude larger than the chiral term inducing the topological phase $\left(t_{2}^{\prime \prime}\right)$. To investigate the onset of the topological phase transition in more detail, we evaluate the spin Chern number of bulk states, defined by $\mathcal{C}_{s}=C_{\uparrow}-C_{\downarrow}$, where $C_{\uparrow}=-C_{\downarrow} \equiv \mathcal{C}$ is the first Chern integer [81]. To compute $\mathcal{C}$ for disordered configurations of adatoms, we employ an efficient gauge-invariant approach developed in Refs. $[82,83]$. This allows us to assess the topological order for realistic TB parameters (i.e., no $t_{n}$ rescaling is required). In Fig. 3(e), we show the dependence of the average Chern number $\overline{\mathcal{C}}$ on the adatom coverage. The robustness of $\overline{\mathcal{C}}$ with respect to $t_{1}^{\prime \prime}$ and its quick suppression at low concentrations when $t_{1}^{\prime}$ and $t_{3}^{\prime}$ are turned on is in perfect agreement with the previous conclusions based on quantum transport simulations in nanoribbon geometry. When all adatom-induced interactions are considered on equal footing, the onset to the transition to a topologically trivial phase is found to occur around 5\% for typical values of the parameters. When approaching $1 \%-2 \%$ coverage, the fluctuations are increasingly larger, predicting 
the closing of the topological gap in the relevant experimental regime. The strong dependence of $\overline{\mathcal{C}}$ on the adatom coverage is reminiscent of Anderson topological insulators [84], for which the character of the insulating ground state is known to critically depend on the disorder strength.

Our findings have several important consequences. The absence of topological gap signatures on recent measurements in adatom-decorated graphene [31-35] is naturally explained by valley mixing processes beyond simple model Hamiltonians. The adatom-induced intervalley scattering uncovered in this Rapid Communication can be mitigated by increasing the spatial range of the interactions mediated by the adsorbate, thereby providing a possible path towards the engineering of a quantum spin Hall insulator in graphene, i.e., its decoration with dilute heavy nanoparticles. These results highlight the importance of seamless multiscale approaches bridging first-principles parametrized model Hamiltonians and large-scale quantum transport calculations for the predictive modeling of adatom-host systems.

The data underlying this Rapid Communication are available from the Figshare database [85].
Acknowledgments. We acknowledge support from the Engineering and Physical Sciences Research Council (EPRSC) under Grants No. EP/N005244/1 (J.L.), No. EP/K003151/1 and No. EP/P023843/1 (K.M.), the Thomas Young Centre under Grant No. TYC-101 (J.L.), the Brazilian funding agency CAPES under Project No. 13703/13-7 (F.J.S.), and the European Research Council (ERC) under the ERC-consolidator Grant No. 681405-DYNASORE (F.J.S.). Via J.L. and K.M.'s membership of the U.K.'s HEC Materials Chemistry Consortium, which is funded by EPSRC (EP/L000202), this work used the ARCHER U.K. National Supercomputing Service. D.A.B. acknowledges the support from Mackpesquisa and FAPESP under Grant No. 2012/50259-8. E.V.C. acknowledges partial support from FCT-Portugal through Grant No. UID/CTM/04540/2013. A.F. gratefully acknowledges the support from the Royal Society through a Royal Society University Research Fellowship and partial funding from EPSRC (Grant No. EP/N004817/1). F.J.S thanks Filipe S. M. Guimarães for support and helpful discussions. E.V.C. and A.F. thank M. P. López-Sancho and M. A. H. Vozmediano for useful discussions.
[1] O. Leenaerts, B. Partoens, and F. M. Peeters, Phys. Rev. B 77, 125416 (2008).

[2] T. Kuila, S. Bose, A. K. Mishra, P. Khanra, N. H. Kim, and J. H. Lee, Prog. Mater. Sci. 57, 1061 (2012).

[3] D. Voiry, A. Goswami, R. Kappera, C. C. C. Silva, D. Kaplan, T. Fujita, M. Chen, T. Asefa, and M. Chhowalla, Nat. Chem. 7, 45 (2015).

[4] C. R. Ryder, J. D. Wood, S. A. Wells, and M. C. Hersam, ACS Nano 10, 3900 (2016).

[5] H. Nan, Z. Wang, W. Wang, Z. Liang, Y. Lu, Q. Chen, D. He, P. Tan, F. Miao, X. Wang, J. Wang, and Z. Ni, ACS Nano 8, 5738 (2014).

[6] S.-H. Cheng, K. Zou, F. Okino, H. R. Gutierrez, A. Gupta, N. Shen, P. C. Eklund, J. O. Sofo, and J. Zhu, Phys. Rev. B 81, 205435 (2010).

[7] R. R. Nair, W. C. Ren, R. Jalil, I. Riaz, V. G. Kravets, L. Britnell, P. Blake, F. Schedin, A. S. Mayorov, S. J. Yuan, M. I. Katsnelson, H. M. Cheng, W. Strupinski, L. G. Bulusheva, A. V. Okotrub, I. V. Grigorieva, A. N. Grigorenko, K. S. Novoselov, and A. K. Geim, Small 6, 2877 (2010).

[8] J. T. Robinson, J. S. Burgess, C. E. Junkermeier, S. C. Badescu, T. L. Reinecke, F. K. Perkins, M. K. Zalalutdniov, J. W. Baldwin, J. C. Culbertson, P. E. Sheehan, and E. S. Snow, Nano Lett. 10, 3001 (2010).

[9] O. Leenaerts, H. Peelaers, A. D. Hernandez-Nieves, B. Partoens, and F. M. Peeters, Phys. Rev. B 82, 195436 (2010).

[10] H. Zhang, E. Bekyarova, J. W. Huang, Z. Zhao, W. Bao, F. Wang, R. C. Haddon, and C. N. Lau, Nano Lett. 11, 4047 (2011).

[11] B. Uchoa, V. N. Kotov, N. M. R. Peres, and A. H. Castro Neto, Phys. Rev. Lett. 101, 026805 (2008).

[12] R. R. Nair, M. Sepioni, I.-L. Tsai, O. Lehtinen, J. Keinonen, A. V. Krasheninnikov, T. Thomson, A. K. Geim, and I. V. Grigorieva, Nat. Phys. 8, 199 (2012).
[13] H. G. Herrero, J. M. G. Rodríguez, P. Mallet, M. Moaied, J. J. Palacios, C. Salgado, M. M. Ugeda, J.-Y. Veuillen, F. Yndurain, and I. Brihuega, Science 352, 437 (2016).

[14] G. Profeta, M. Calandra, and F. Mauri, Nat. Phys. 8, 131 (2012).

[15] K. Li, X. Feng, W. Zhang, Y. Ou, L. Chen, K. He, L.-L. Wang, L. Guo, G. Liu, Q.-K. Xue, and X. Ma, Appl. Phys. Lett. 103, 062601 (2013).

[16] B. M. Ludbrook, G. Levy, P. Nigge, M. Zonno, M. Schneider, D. J. Dvorak, C. N. Veenstra, S. Zhdanovich, D. Wong, P. Dosanjh, C. Straßer, A. Stöhr, S. Forti, C. R. Ast, U. Starke, and A. Damascelli, Proc. Natl Acad. Sci. USA 112, 11795 (2015).

[17] J. Chapman, Y. Su, C. A. Howard, D. Kundys, A. N. Grigorenko, F. Guinea, A. K. Geim, I. V. Grigorieva, and R. R. Nair, Sci. Rep. 6, 23254 (2016).

[18] D. Huertas-Hernando, F. Guinea, and A. Brataas, Phys. Rev. Lett. 103, 146801 (2009).

[19] K. Pi, W. Han, K. M. McCreary, A. G. Swartz, Y. Li, and R. K. Kawakami, Phys. Rev. Lett. 104, 187201 (2010).

[20] D. V. Fedorov, M. Gradhand, S. Ostanin, I. V. Maznichenko, A. Ernst, J. Fabian, and I. Mertig, Phys. Rev. Lett. 110, 156602 (2013).

[21] D. V. Tuan, F. Ortmann, D. Soriano, S. O. Valenzuela, and S. Roche, Nat. Phys. 10, 857 (2014).

[22] A. Pachoud, A. Ferreira, B. Ozyilmaz, and A. H. Castro Neto, Phys. Rev. B 90, 035444 (2014).

[23] A. Ferreira, T. G. Rappoport, M. A. Cazalilla, and A. H. Castro Neto, Phys. Rev. Lett. 112, 066601 (2014).

[24] C. Huang, Y. D. Chong, and M. A. Cazalilla, Phys. Rev. B 94, 085414 (2016).

[25] J. Balakrishnan, G. K. W. Koon, A. Avsar, Y. Ho, J. H. Lee, M. Jaiswal, S.-J. Baeck, J.-H. Ahn, A. Ferreira, M. A. Cazalilla, A. H. Castro Neto, and B. Ozyilmaz, Nat. Commun. 5, 4748 (2014). 
[26] C. Weeks, J. Hu, J. Alicea, M. Franz, and R. Wu, Phys. Rev. X 1, 021001 (2011).

[27] H. Zhang, C. Lazo, S. Blügel, S. Heinze, and Y. Mokrousov, Phys. Rev. Lett. 108, 056802 (2012).

[28] H. Jiang, Z. Qiao, H. Liu, J. Shi, and Q. Niu, Phys. Rev. Lett. 109, 116803 (2012).

[29] L. Brey, Phys. Rev. B 92, 235444 (2015).

[30] C. L. Kane and E. J. Mele, Phys. Rev. Lett. 95, 226801 (2005).

[31] U. Chandni, E. A. Henriksen, and J. P. Eisenstein, Phys. Rev. B 91, 245402 (2015).

[32] Y. Wang, S. Xiao, X. Cai, W. Bao, J. R. Robey, and M. S. Fuhrer, Sci. Rep. 5, 15764 (2015).

[33] Z. Jia, B. Yan, J. Niu, Q. Han, R. Zhu, D. Yu, and X. Wu, Phys. Rev. B 91, 085411 (2015).

[34] Y. Wang, X. Cai, J. Reutt-Robey, and M. S. Fuhrer, Phys. Rev. B 92, 161411(R) (2015).

[35] J. A. Elias and E. A. Henriksen, Phys. Rev. B 95, 075405 (2017).

[36] A. Cresti, D. Van Tuan, D. Soriano, A. W. Cummings, and S. Roche, Phys. Rev. Lett. 113, 246603 (2014).

[37] D. Huertas-Hernando, F. Guinea, and A. Brataas, Phys. Rev. B 74, 155426 (2006).

[38] H. Min, J. E. Hill, N. A. Sinitsyn, B. R. Sahu, L. Kleinman, and A. H. MacDonald, Phys. Rev. B 74, 165310 (2006).

[39] S. Konschuh, M. Gmitra, and J. Fabian, Phys. Rev. B 82, 245412 (2010).

[40] J. Sichau, M. Prada, T. J. Lyon, B. Bosnjak, L. Tiemann, and R. H. Blick, arXiv:1709.05705.

[41] M. König, S. Wiedmann, C. Brüne, A. Roth, H. Buhmann, L. W. Molenkamp, X.-L. Qi, and S.-C. Zhang, Science 318, 766 (2007).

[42] C. Liu, T. L. Hughes, X.-L. Qi, K. Wang, and S.-C. Zhang, Phys. Rev. Lett. 100, 236601 (2008).

[43] L. Du, I. Knez, G. Sullivan, and R.-R. Du, Phys. Rev. Lett. 114, 096802 (2015).

[44] See Supplemental Material at http://link.aps.org/supplemental/ 10.1103/PhysRevB.98.081407 for methodology, derivation of effective Hamiltonian, and additional quantum transport and topological invariant calculations, which includes Refs. [45-65].

[45] P. Giannozzi et al., J. Phys.: Condens. Matter 21, 395502 (2009).

[46] D. R. Hamann, Phys. Rev. B 88, 085117 (2013).

[47] J. P. Perdew, K. Burke, and M. Ernzerhof, Phys. Rev. Lett. 77, 3865 (1996).

[48] H. J. Monkhorst and J. D. Pack, Phys. Rev. B 13, 5188 (1976).

[49] R. C. Weast and M. J. Astle, CRC Handbook of Chemistry and Physics (CRC Press, Boca Raton, FL, 1982).

[50] M. S. Hybertsen and S. G. Louie, Phys. Rev. B 34, 5390 (1986).

[51] J. Deslippe, G. Samsonidze, D. A. Strubbe, M. Jain, M. L. Cohen, and S. G. Louie, Comput. Phys. Commun. 183, 1269 (2012).

[52] J. Deslippe, G. Samsonidze, M. Jain, M. L. Cohen, and S. G. Louie, Phys. Rev. B 87, 165124 (2013).

[53] A. A. Mostofi, J. R. Yates, Y.-S. Lee, I. Souza, D. Vanderbilt, and N. Marzari, Comput. Phys. Commun. 178, 685 (2008).

[54] N. Marzari and D. Vanderbilt, Phys. Rev. B 56, 12847 (1997).

[55] I. Souza, N. Marzari, and D. Vanderbilt, Phys. Rev. B 65, 035109 (2001).
[56] J. Jung and A. H. MacDonald, Phys. Rev. B 87, 195450 (2013).

[57] Y.-W. Son, M. L. Cohen, and S. G. Louie, Phys. Rev. Lett. 97, 216803 (2006).

[58] H. Zheng, Z. F. Wang, T. Luo, Q. W. Shi, and J. Chen, Phys. Rev. B 75, 165414 (2007).

[59] M. Büttiker, Phys. Rev. B 38, 9375 (1988).

[60] R. Landauer, Philos. Mag. 21, 863 (1970).

[61] C. Caroli, R. Combescot, P. Nozieres, and D. Saint-James, J. Phys. C: Solid State Phys. 4, 916 (1971).

[62] S. Datta, Electronic Transport in Mesoscopic Systems (Cambridge University Press, Cambridge, U.K., 1995).

[63] D. S. Fisher and P. A. Lee, Phys. Rev. B 23, 6851 (1981).

[64] C. H. Lewenkopf and E. R. Mucciolo, J Comput. Electron. 12, 203 (2013).

[65] H. Haug and A.-P. Jauho, Quantum Kinetics in Transport and Optics of Semiconductors, 2nd ed. (Springer, Berlin, 2007).

[66] Spin-dependent tunneling processes (e.g., $\propto \Omega_{m, \uparrow}^{\dagger} d_{m, \downarrow}$ ) have a negligible probability and thus are omitted. Moreover, crystalfield effects are neglected [26,44,67].

[67] T. P. Cysne, A. Ferreira, and T. G. Rappoport, Phys. Rev. B 98, 045407 (2018).

[68] C. Bena, Phys. Rev. Lett. 100, 076601 (2008).

[69] The low-energy theory for generic physisorbed adatoms was first derived in Ref. [22].

[70] M. Z. Hasan and C. L. Kane, Rev. Mod. Phys. 82, 3045 (2010).

[71] E. Fradkin, Phys. Rev. B 33, 3257 (1986); 33, 3263 (1986).

[72] H. Suzuura and T. Ando, Phys. Rev. Lett. 89, 266603 (2002).

[73] A. Roth, C. Brüne, H. Buhmann, L. W. Molenkamp, J. Maciejko, X.-L. Qi, and S.-C. Zhang, Science 325, 294 (2009).

[74] V. M. Pereira, F. Guinea, J. M. B. Lopes dos Santos, N. M. R. Peres, and A. H. Castro Neto, Phys. Rev. Lett. 96, 036801 (2006).

[75] M. M. Ugeda, I. Brihuega, F. Guinea, and J. M. GomezRodriguez, Phys. Rev. Lett. 104, 096804 (2010).

[76] V. Häfner, J. Schindler, N. Weik, T. Mayer, S. Balakrishnan, R. Narayanan, S. Bera, and F. Evers, Phys. Rev. Lett. 113, 186802 (2014).

[77] A. Ferreira and E. R. Mucciolo, Phys. Rev. Lett. 115, 106601 (2015).

[78] N. Weik, Phys. Rev. B 94, 064204 (2016).

[79] H.-Y. Deng and K. Wakabayashi, Phys. Rev. B 91, 035425 (2015).

[80] J.-Y. Yan, P. Zhang, B. Sun, H.-Z. Lu, Z. Wang, S. Duan, and X. G. Zhao, Phys. Rev. B 79, 115403 (2009).

[81] D. N. Sheng, Z. Y. Weng, L. Sheng, and F. D. M. Haldane, Phys. Rev. Lett. 97, 036808 (2006).

[82] T. Fukui, Y. Hatsugai, and H. Suzuki, J. Phys. Soc. Jpn. 74, 1674 (2005).

[83] Y. F. Zhang, Y. Y. Yang, Y. Ju, L. Sheng, D. N. Sheng, R. Shen, and D. Y. Xing, Chin. Phys. B 22, 117312 (2013).

[84] J. Li, R.-L. Chu, J. K. Jain, and S.-Q. Shen, Phys. Rev. Lett. 102, 136806 (2009).

[85] doi: https://doi.org/10.6084/m9.figshare.6934883.v1 